\title{
REGIME DE RADIAÇÃO SOLAR E CARACTERÍSTICAS DA VEGETAÇÃO - MODELOS DE INVERSÃO.
}

\author{
Arí de O. MARQUES FILHO
}

RESUMO - Um tratamento do regime de radiação no interior de florestas em conexão com a inversão de modelos matemáticos para estimar as características da vegetação (densidade de área foliar, LAI, etc.) é apresentado e discutido neste estudo. Medidas de radiação solar (PAR) realizadas no periodo de janeiro a abril de 1994 na Reserva Florestal Ducke (Manaus, AM) foram utilizadas para testar o algoritmo resultante e a distribuição vertical de área foliar para a vegetação local foi estabelecida.

Palavras-chaves: radiação solar, modelos de inversão, área foliar, floresta.

Solar Radiation Regime and Characteristics of the Vegetation - Inversion Models.

ABSTRACT - This paper presents a method in which a mathematical model of the solar radiation regime below the canopy of a tropical rainforest is inverted to estimate the vegetation characteristics (foliar area density, leaf area index, LAI, etc.). Solar radiation measurements (PAR) made in the period from january to april 1994 at the Reserva Florestal Ducke (Manaus - AM) were used to test the algorithm which was created and the foliar area vertical distribution from the local vegetation was established.

Keywords: Solar radiation, inversion models, foliar area, forest.

\section{INTRODUÇÃO}

A caracterização de uma cobertura vegetal em sua estrutura e distribuição espacial dos elementos vegetais representa o ponto de partida para o estudo de fenômenos físicos e fisiológicos que ocorrem em seu interior. Em coberturas vegetais de pequeno e médio porte essa fase inicial descritiva recebe o suporte de métodos (diretos ou indiretos) já bem consolidados de medidas das características principais da vegetação. No caso de florestas cobertura vegetal de grande porte - os procedimentos são bem delicados e de operacionalidade rica em dificuldades: os métodos diretos não são aplicáveis em geral; os métodos indiretos que se baseiam em medidas de radiação no interior e acima da cobertura recebem atenção especial e assumem o papel central, mesmo que retenham dificuldades importantes e exijam equipamentos especiais para as medidas na parte superior da vegetação. ANDRIEU \& BARET (1993) apresentam uma revisão detalhada sobre os métodos indiretos de estimar a estrutura da cobertura a partir de medidas de luz em seu interior. ROSS (1981) discute os aspectos gerais da distribuição e orientação espacial dos elementos vegetais, formando uma base teórica sobre a qual repousa o tema central de seu trabalho: o regime de radiação em meio vegetal. MYNENI et al (1989) apresentam uma revisão suficientemente ampla sobre a radiação solar no meio végetal, ressaltando os aspectos numéricos apropriados para o desenvolvimento dos diferentes modelos e de suas soluções. A quantidade e a forma de

1 Instituto Nacional de Pesquisas da Amazonia Manaus - AM 
orientação no espaço dos elementos vegetais em cada zona (posições horizontais e verticais) são reflexo da estrutura, determinam a densidade de uma cobertura e constituem os fatores primários não só no regime de radiação como também nas trocas gasosas e energéticas entre o meio vegetal e a atmosfera. No caso específico da radiação solar no interior de vegetação densa torna-se necessário o conhecimento prévio do total de área foliar existente entre o solo e o topo da cobertura (LAI - leaf area index) e de sua distribuição na vertical (descrição unidimensional somente) para a definição dos perfis de radiação e a partir daí inferir a distribuição de energia de natureza radiativa nas diferentes camadas horizontais da vegetação.

Nesse contexto, o objetivo primário deste estudo é a caracterização da vegetação densidade de área foliar e o LAI - a partir de medidas de perfis de radiação solar ( radiação fotossinteticamente ativa, PAR) em seu interior em estreita associação com procedimentos matemáticos de inversão de modelos teóricos pertinentes. As medidas de radiação usadas nesta análise foram realizadas no período de janeiro a abril de 1994 no sitio experimental da Reserva Florestal Ducke, Manaus - AM.

\section{RADIAÇÃO SOLAR EM MEIO VEGETAL E INVERSÃO DE MODELOS}

$\mathrm{O}$ regime de radiação solar no interior de uma cobertura vegetal resulta de uma série de interações entre a radiação incidente e os elementos vegetais, especialmente as folhas com suas características óticas (reflexão e transmissão), orientação e distribuição espacial. O total de radiação numa altura $\mathrm{z}$ no interior da cobertura vegetal pode ser separado em parcelas associadas aos campos primário e complementar de radiação direta e difusa, num total de quatro componentes de incidência sobre a face superior de uma superficie horizontal de referência (fluxo descendente) e duas componentes de incidência sobre a face inferior dessa referência (fluxo ascendente); os reduzidos valores relativos dos fluxos ascendentes - o albedo de uma cobertura vegetal não ultrapassa $20 \%$ e o fluxo ascendente máximo não ultrapassa portanto $20 \%$ da radiação incidente - não favorece a sua utilização como modelo de inversão para inferir características da vegetação. Com esse argumento seria suficiente detalhar-se modelos analíticos para as quatro componentes do fluxo descendente; entretanto, a descrição do campo complementar vincula esses fluxos e a sua separação torna-se possível somente após o desenvolvimento das soluções correspondentes. Assim, as componentes dos campos primário e complementar de radiação direta e difusa são descritas pelas seguintes equações (MARQUES FILHO, 1992):

\section{Radiação direta primária}

$$
F_{s}(z)=F_{s}(h) \cdot e^{-K\left(\theta_{s}\right) A(z)}
$$

\section{Radiação difusa primária}

$$
F_{d}(z)=F_{d}(h) \int_{0}^{\pi / 2} e^{-K(\theta) /(z)} \cos \theta \cdot \operatorname{sen} \theta \cdot \mathrm{d} \theta
$$




\section{Radiação Complementar (direta ou difusa)}

Fluxo descendente

$$
F_{1}(A)=F_{p 1}(A)-\frac{\Phi\left[\mu, r\left(A_{0}-A\right)\right]-\alpha_{s} \Phi\left[1, r\left(A_{0}-A\right)\right]}{\Phi\left[\mu, r A_{0}\right]-\alpha_{s} \Phi\left[1, r A_{0}\right]} F_{p 1}(0)-\frac{F_{p 2}\left(A_{0}\right)-\alpha_{s}\left\{F\left(A_{0}\right)+F_{p 1}\left(A_{0}\right)\right\}}{\Phi\left[\mu, r A_{0}\right]-\alpha_{s} \Phi\left[1, r A_{0}\right]} \Phi[1, r A]
$$

Fluxo ascendente

$$
F_{2}(A)=F_{p 2}(A)-\frac{\Phi\left[1, r\left(A_{0}-A\right)\right]-\alpha_{s} \Phi\left[1 / \mu, r\left(A_{0}-A\right)\right]}{\Phi\left[\mu, r A_{0}\right]-\alpha_{s} \Phi\left[1 . r A_{0}\right]} F_{p 1}(0)-\frac{F_{p 2}\left(A_{0}\right)-\alpha_{s}\left\{F\left(A_{0}\right)+F_{p 1}\left(A_{0}\right)\right\}}{\Phi\left[\mu, r A_{0}\right]-\alpha_{s} \Phi\left[1, r A_{0}\right]} \Phi[\mu, r A]
$$

onde,

$\mathrm{F}(\mathrm{z})=$ fluxo de radiação (direta, difusa ou complementar) no nível z no interior da vegetação (W. $\mathrm{m}^{-2}$ ).

$\mathrm{F}(\mathrm{h})=$ fluxo de radiação (direta ou difusa) no topo da vegetação $\left(\mathrm{W}_{\mathrm{m}^{-2}}\right)$

$\alpha_{\mathrm{s}}=$ albedo do solo.

$\mathrm{A}(\mathrm{z})$ = área foliar acumulada (adimensional)

$\mathrm{A}_{0}=$ área foliar acumulada total (LAI da vegetação)

$r, \mu=$ parâmetros dependentes dos coeficientes de reflexão e transmissão dos elementos vegetais $(\mathrm{R}$ e $\mathrm{T})$.

$K(\theta)=$ coeficiente de atenuação $(=1 / 2 \cos \theta)$ para a orientação aleatória dos elementos vegetais

$$
\Phi[x, y]=x e^{y}-\left(x e^{y}\right)^{-1}
$$

As principais hipóteses implicitas no desenvolvimento dessas equações são as seguintes:

- distribuição espacial aleatória dos elementos vegetais;

- orientação aleatória dos elementos vegetais;

- isotropia do campo de radiação difusa (primária e complementar).

Essas equações expressam as soluções para as diferentes componentes de radiação em condições suficientemente gerais. Numa abordagem que trata sobre a inversão de modelos é conveniente e possivel trabalhar-se num domínio de soluções mais simples, que é definido em função dos instrumentos de medidas da radiação e das dificuldades amostrais para se obter uma medida representativa em cada zona da cobertura vegetal.

Parece natural assumir-se que o campo de radiação difusa no interior de coberturas vegetais é fracamente dependente das peculiaridades estruturais e da distribuição espacial dos seus elementos; as dificuldades amostrais das medidas devem ser associadas substancialmente às componentes da radiação direta, que determinam em cada nivel um campo heterogêneo compativel com as irregularidades inerentes à cobertura. Então, no conjunto de equações apresentado acima focaliza-se apenas os termos de radiação difusa e sua adaptação para o procedimento de inversão.

As formas das funções $\mathrm{F}_{\mathrm{p} 1}$ e $\mathrm{F}_{\mathrm{p} 2}$ são mostradas nas fĩguras 1 e 2 em função de $\mathrm{A}(\mathrm{z})$ (área foliar acumulada) e de $(\mathrm{R}+\mathrm{T})$ que representa o espalhamento da rádiação (Reflexões e Transmissões). Tais funções governam o campo complementar da radiação difusa em sua maior parte, dependem do espalhamento da radiação na vegetação e são descritas pela equação $(\mathrm{k}=1,2)$ : 


$$
F_{p k}(A)=\sum_{j=1}^{3}\left[\kappa_{j, k} E_{j}\left(\frac{A}{2}\right)\right]+\kappa_{4, k} e^{-r A} E_{1}^{*}\left(\frac{(1-2 r) A}{2}\right)+\kappa_{5, k} e^{r A} E_{1}\left(\frac{(1-2 r) A}{2}\right)
$$

onde,

$$
E_{n}(x)=\int_{1}^{\infty} \frac{e^{-x t}}{t^{n}} d t, \quad E_{1}^{*}(x)=\int_{x}^{\infty} \frac{e^{-t}}{t} d t \quad \text { e } \quad A(z)=\int_{2}^{h} a(z) d z .
$$

a(z) densidade de área foliar $\left(\mathrm{m}^{2} / \mathrm{m}^{3}\right)$.

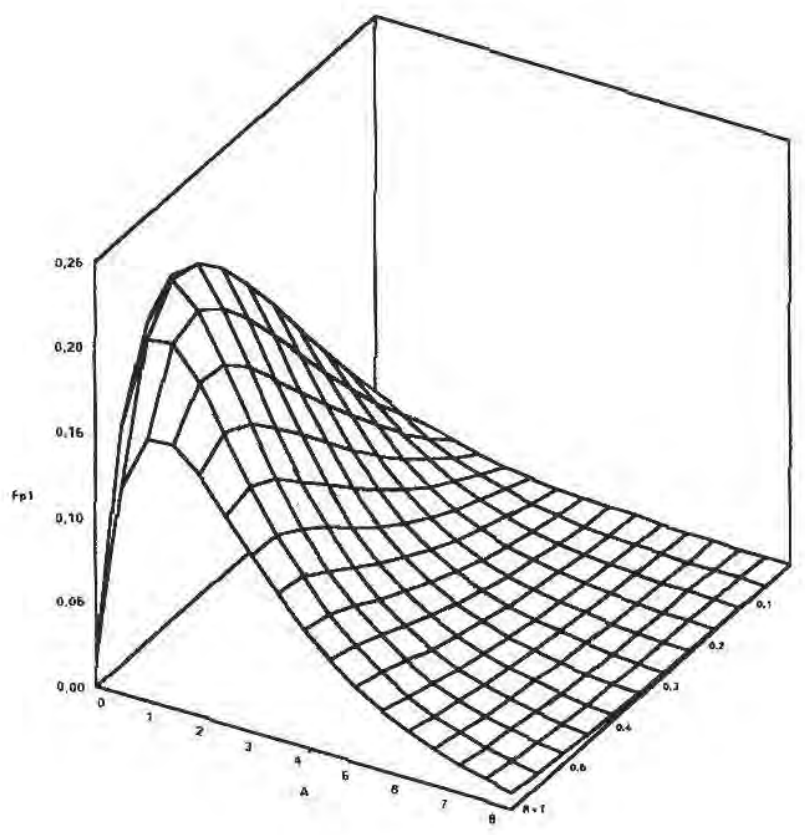

Figura 1. Função $F_{p 1}$ e sua relação com o espalhamento e a área foliar acumulada. 


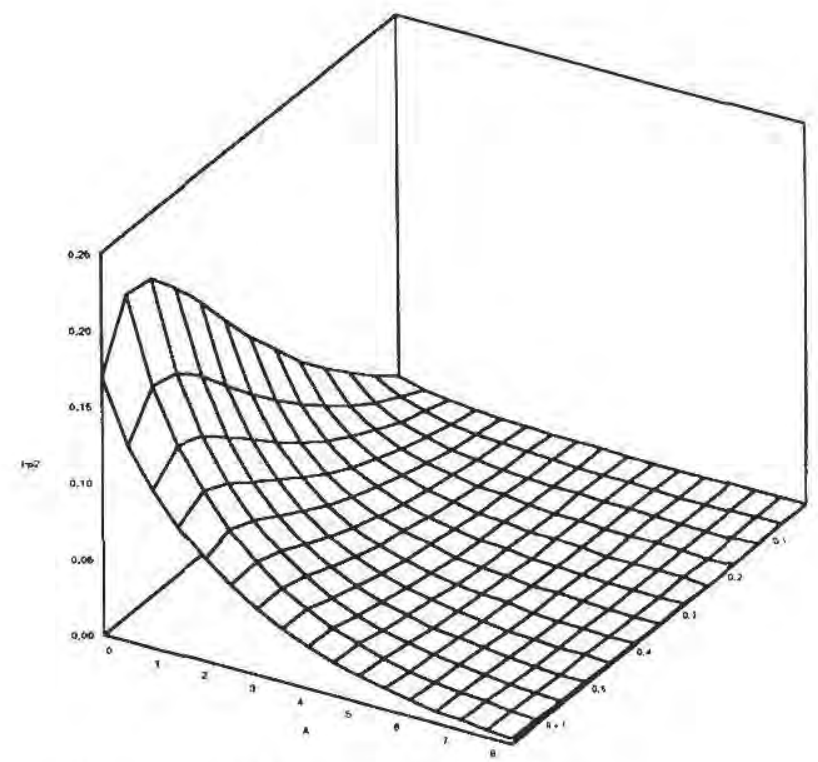

Figura 2. Função $\mathrm{F}_{\mathrm{p} 2}$ e sua relação com o espalhamento.

Nessas figuras, as funções $\mathrm{F}_{\mathrm{pl}} \mathrm{e}$ $\mathrm{F}_{\mathrm{p} 2}$, núcleos geradores dos fluxos de radiação complementar, apresentam uma conformação regular decrescente na região de baixos valores de espalhamento $(R+T=0,3)$; para valores mais altos de espalhamento o comportamento é bem mais complexo, e a função $F_{p l}$ passa a apresentar um valor máximo na zona próxima ao topo da vegetação.
O espalhamento reduzido de radiação PAR $(R+T=0,15)$ permite simplificações nas equações de definição ( 3 e 4 ) em função dos parâmetros $\mu=(1$ $\left.\mathrm{W}_{\mathrm{a}}+\mathrm{r}\right) / \mathrm{W}_{\mathrm{b}}, \mathrm{r}$ e $\mathrm{A}_{0}$. $\mathrm{O}$ albedo do solo para vegetações densas pode também ser negligenciado $\left(\alpha_{s}=0\right)$. Essas modificações não alteram em termos práticos as curvas resultantes e facilitam a tarefa da inversão, como pode ser constatado nas formas das equações seguintes:

$$
\begin{gathered}
F_{1}(A)=F_{p 1}(A)-e^{-r A} F_{p 1}(0)-\frac{e^{-r\left(A_{0}-A\right)}\left\{1-e^{-2 r A}\right\}}{\mu} F_{p 2}\left(A_{0}\right) \\
F_{2}(A)=F_{p 2}(A)-e^{-r A}\left\{\frac{1-e^{-2 r\left(A_{0}-A\right)}}{\mu}\right\} F_{p 1}(0)-e^{-r\left(A_{0}-A\right)} F_{p 2}\left(A_{0}\right)
\end{gathered}
$$


Essas duas expressões facilitam também a tarefa de identificar tendências para cada um dos fluxos complementares. Nas relações mostradas abaixo as tendências preconizadas são tanto mais verdadeiras quanto maior for o valor do parâmetro $\mu$ e devem persistir mesmo quando o caso geral expresso pelas equações 3 e 4 for considerado.

$$
\begin{aligned}
& A \rightarrow A_{0} \Leftrightarrow F_{1}(A) \equiv F_{p 1}(A) \\
& A \rightarrow 0 \Leftrightarrow F_{2}(A) \equiv F_{p 2}(A)
\end{aligned}
$$

Os fluxos descendentes de radiação, somados os campos primários e complementares, são mais adequados para estudos de inversão de modelos por apresentarem magnitudes bem superiores àquelas dos fluxos ascendentes. Juntando $a$ isso a restrição anterior sobre o uso de medidas de radiação direta para a inversão de modelos, resultam dois focos de interesse nas expressões anteriores como elementos a serem trabalhados no procedimento de inversão: a equação 2 (campo primário) e a equação 6 (campo complementar de radiação difusa). Assumindo-se $\mathrm{R}=\mathrm{T}=0,075$ (espalhamento total igual a 0,15 ) como valores padrões para a radiação PAR, uma visão relativa dos diferentes fluxos e funções é apresentada na tabela 1 . Os fluxos relativos e os parâmetros re $\mu$ são definidos pelas seguintes relações $(\mathrm{F}(0)=$ radiação global no topo da vegetação):
Na tabela 1, o fluxo relativo $\alpha_{1}$ está ligado diretamente à área foliar acumulada A por funções matemáticas conhecidas; 0 problema inverso que está sendo tratado neste estudo consiste em determinar a função $\mathrm{A}(\mathrm{z})$ (ou a densidade de área foliar, a(z)) com o suporte de medidas de perfis de radiação solar, estabelecendo previamente a função inversa que liga as duas variáveis (inversão analítica), ou então, criando um algoritmo computacional que realize a operação de inversão. A natureza das funções envolvidas não justifica o esforço de análise necessário para explicitar a função inversa na primeira alternativa; o algoritmo numérico na segunda alternativa é em princípio iterativo ou dependente de um conhecimento prévio do LAI da cobertura vegetal. Entretanto, observando-se os valores da função $\mathrm{F}_{\mathrm{p} 2}$ (A) na tabela 1 e a ordem de grandeza do parâmetro $\mu(\sim 25)$ para a radiação PAR, conclui-se que para essas condições o fluxo complementar descendente é independente de $\mathrm{A}_{0}$. O problema de inversão de modelo resume-se na busca da solução de uma equação implícita que gera um valor preciso de $\mathrm{A}(\mathrm{z})$ para cada valor medido de $\alpha$. Na tabela 2 são apresentados em forma tabular os valores de $\mathrm{A}(\mathrm{z})$ (precisão decimal) que resultam dos diferentes valores supostamente medidos de fluxo relativo $\alpha_{t}$ (corpo da tabela). Valores intermediários que não constam na tabela podem ser interpolados ou calculados exatamente pelas formulações discutidas anteriormente.

$$
\begin{array}{ll}
\alpha_{d}(A)=F_{d}(A) / F(0) & \alpha_{1}(A)=F_{1}(A) / F(0) \quad \alpha_{1}(A)=\left[F_{d}(A)+F_{1}(A)\right] / F(0) \\
r=\sqrt{1-R-T} & \mu=(1-R+r) / T
\end{array}
$$


Tabela 1. Fluxos de Radiação difusa

\begin{tabular}{cccccc}
\hline $\mathrm{A}$ & $\mathrm{F}_{\mathrm{p} 1}$ & $\mathrm{~F}_{\mathrm{p} 2}$ & $\alpha_{1}$ & $\alpha_{\mathrm{d}}$ & $\alpha_{\mathrm{t}}$ \\
\hline 0 & 0,078 & 0,040 & 0,000 & 1,000 & 1,000 \\
1 & 0,057 & 0,017 & 0,026 & 0,443 & 0,469 \\
2 & 0,034 & 0,008 & 0,021 & 0,219 & 0,240 \\
3 & 0,019 & 0,004 & 0,014 & 0,113 & 0,127 \\
4 & 0,010 & 0,002 & 0,008 & 0,060 & 0,068 \\
5 & 0,005 & 0,001 & 0,005 & 0,033 & 0,038 \\
6 & 0,003 & 0,001 & 0,003 & 0,018 & 0,021 \\
7 & 0,002 & 0,000 & 0,002 & 0,010 & 0,012 \\
8 & 0,001 & 0,000 & 0,001 & 0,006 & 0,007 \\
\hline
\end{tabular}

Tabela 2. Relação tabulada de $A=A\left(\alpha_{1}\right)$

\begin{tabular}{c|cccccccccc}
\hline $\mathrm{A}$ & 0,0 & 0,1 & 0,2 & 0,3 & 0,4 & 0,5 & 0,6 & 0,7 & 0,8 & 0,9 \\
\hline 0 & 1,000 & 0,917 & 0,845 & 0,781 & 0,723 & 0,670 & 0,623 & 0,580 & 0,540 & 0,503 \\
1 & 0,469 & 0,438 & 0,409 & 0,383 & 0,357 & 0,335 & 0,313 & 0,293 & 0,274 & 0,257 \\
2 & 0,240 & 0,225 & 0,212 & 0,198 & 0,186 & 0,174 & 0,163 & 0,154 & 0,144 & 0,135 \\
3 & 0,127 & 0,119 & 0,112 & 0,106 & 0,099 & 0,093 & 0,087 & 0,082 & 0,077 & 0,072 \\
4 & 0,068 & 0,065 & 0,060 & 0,057 & 0,053 & 0,050 & 0,048 & 0,044 & 0,042 & 0,040 \\
5 & 0,038 & 0,035 & 0,033 & 0,031 & 0,030 & 0,027 & 0,026 & 0,024 & 0,023 & 0,022 \\
6 & 0,021 & 0,020 & 0,019 & 0,017 & 0,016 & 0,015 & 0,015 & 0,014 & 0,013 & 0,012 \\
7 & 0,012 & 0,011 & 0,010 & 0,009 & 0,009 & 0,008 & 0,008 & 0,008 & 0,007 & 0,007 \\
8 & 0,007 & 0,006 & 0,006 & 0,006 & 0,005 & 0,005 & 0,005 & 0,005 & 0,004 & 0,003 \\
\hline
\end{tabular}

\section{RESULTADOS OBTIDOS E DISCUSSÃO}

As medidas de radiação PAR (400-700 nm) utilizadas nesta análise foram realizadas na Reserva Florestal Ducke (Manaus - AM) no período de 19 de janeiro a 13 de abril de 1994, com o suporte da torre metálica ali existente para a distribuição dos sensores em diferentes alturas no interior da vegetação.

O sistema de medidas está composto por 18 sensores de radiação (modelo SKP 215 - SKYE INSTRUMENTS) acoplados a um logger com multiplexador (modelo 21x - Campbell Instruments), que facilita a leitura de um grande número de sensores com adequada definição temporal das medidas. Os sensores foram calibrados para gerar uma medida de fluxo de energia $\left(\mathrm{Watt} / \mathrm{m}^{2}\right)$ para os planos onde eles foram instalados. Os coeficientes de calibração variam numa pequena faixa de valores: 20 a $27 \mathrm{Watt} / \mathrm{m}^{2}$ para 1 milivolt.

Em cada vertical, um grupo de três sensores foi adaptado sobre um tubo metálico de $6 \mathrm{~m}$ de comprimento que os mantinha afastados da lateral da torre a uma distância média de $4 \mathrm{~m}$; em cada grupo os sensores foram ajustados a um mesmo plano horizontal e forneciam três medidas para o cálculo de um fluxo de radiação descendente médio para o nível considerado. Os três primeiros 
instrumentos foram instalados a uma altura de $8,23 \mathrm{~m}$ do solo ( 27 pés), e a partir desse nível para o alto os outros conjuntos foram colocados a cada 5,48 $\mathrm{m}$ (18 pés); os níveis de medidas assim definidos são os seguintes: $8,23 \mathrm{~m}(27$ pés); 13,72 m (45 pes); 19,2m (63 pés); $24,69 \mathrm{~m}$ ( 81 pés); $30,18 \mathrm{~m}$ (99 pés); $38 \mathrm{~m}$ (topo da vegetação).

O sistema de aquisição de dados foi programado para fazer uma varredura nos canais de entrada a cada 10 segundos no período diurno das $6: 00 \mathrm{~h}$ às $18: 00$, compondo a partir dessas medidas os valores médios para intervalos de 20 minutos.

\section{Reserva Ducke - 12/02/94}

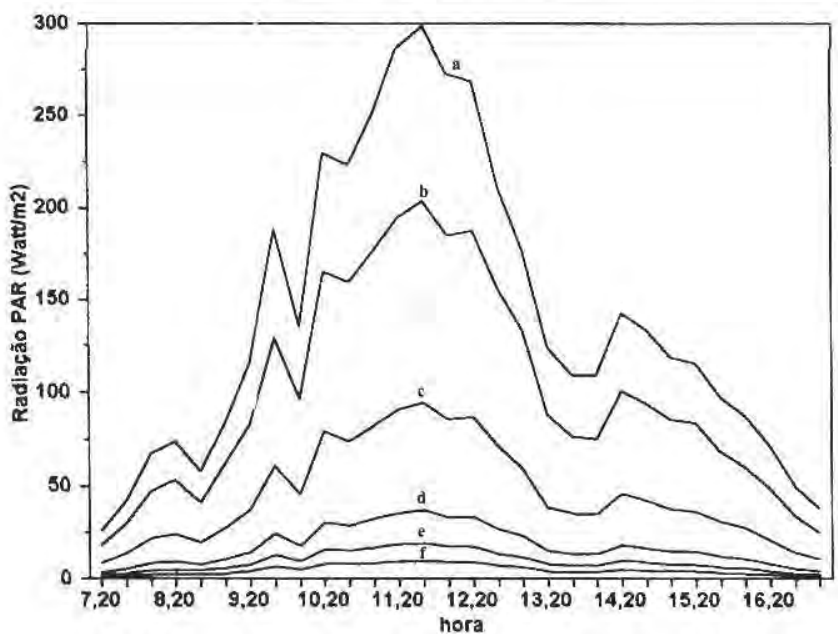

Figura 3. Variação temporal da radiação PAR (12/02/94).

Reserva Ducke 27/02/94

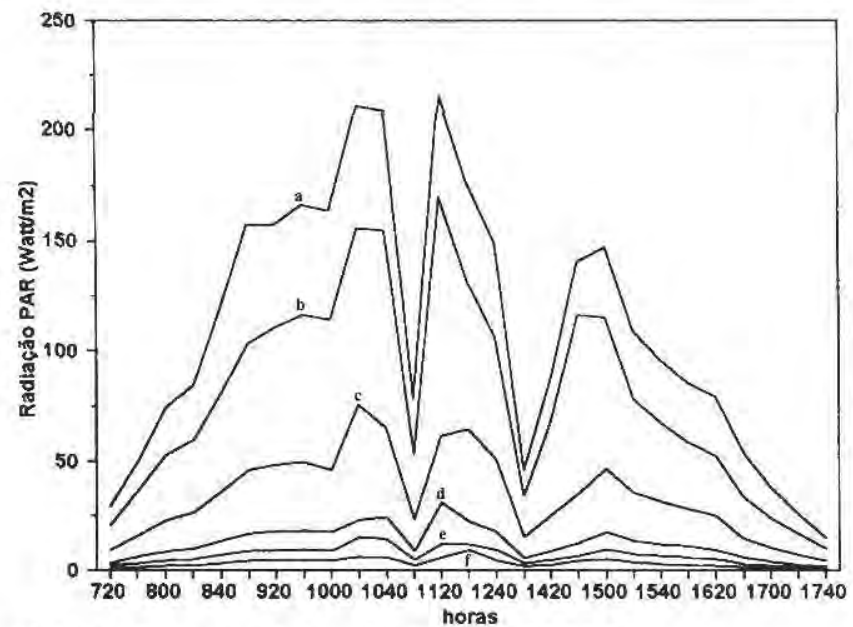

Figura 4. Variação temporal da radiação PAR (27/02/94) 
A figura 3 ilustra a variação temporal da radiação PAR acima e no interior da vegetação para o dia (com moderada cobertura de nuvens) 12/02/94 e a figura 4 exemplifica as condições de radiação para um dia com céu encoberto.

A consistência das medidas pode ser avaliada pela similaridade qualitativa das curvas desde o topo da floresta até o nível mais próximo do solo nos dois dias apresentados. Esse fato pode ser explicado pela forte dominância de radiação difusa para os dias que apresentam o céu parcialmente encoberto.

A predominância de radiação difusa num determinado local para um dado período do ano está associada à cobertura de nuvens, uma característica da atmosfera de difícil previsão e cuja formação pode estar associada à múltiplas fontes atuando de forma isolada ou combinada. Para o objetivo central do presente estudo é suficiente uma avaliação relativa da existência e regularidade (distribuição espacial aproximadamente homogênea) de coberturas de nuvens; isto pode ser inferido indiretamente se o padrão horário đe radiação solar global para céu limpo for conhecido ou observado. Num dado período do ano esse padrão pode ser estimado a partir dos valores de radiação solar máximos observados em cada horário. Na figura 5 esses valores máximos para o período de observação (19/01 - 20/ 04/94) são apresentados juntamente com a curva envoltória ajustada, que representa para esse periodo e local a radiação solar global (PAR) máxima possível.

Conhecida a curva horária de radiação máxima possível, torna-se disponivel um referencial para separar e classificar os perfis observados, tomando-se a razão entre a radiação observada no topo da cobertura em cada perfil particular e a radiação máxima estimada para o horário em questão como critério de classificação. A contribuição da radiação direta para os fluxos medidos no topo e também no interior da vegetaçã̃o diminui com o aumento da cobertura de nuvens. Em termos de modelização e a sua complexidade associada com a direcionalidade da radiação direta o efeito dessa contribuição deve ser marginal já para valores da ordem de $50 \%$

Radiaçăo PAR máxima

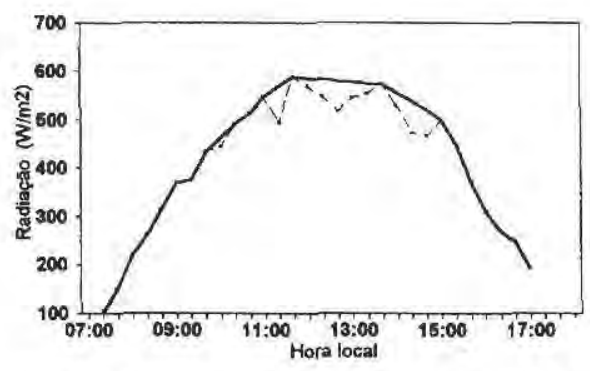

Figura 5. Radiação solar global (PAR) máxima estimada .

da radiação máxima. Em correspondência, para essas condiçōes os valores medidos no interior da vegetação são menos afetados pelas limitações de amostragem espacial inerentes em coberturas vegetais de grande porte: $o$ campo de radiação é predominantemente isotrópico e um modelo de radiação difusa é suficiente para representá-lo.

Os perfis observados foram normalizados pela radiação medida no topo da floresta e separados em cinco categorias conforme o resultado da razão entre essa radiação e a máxima possível para o 

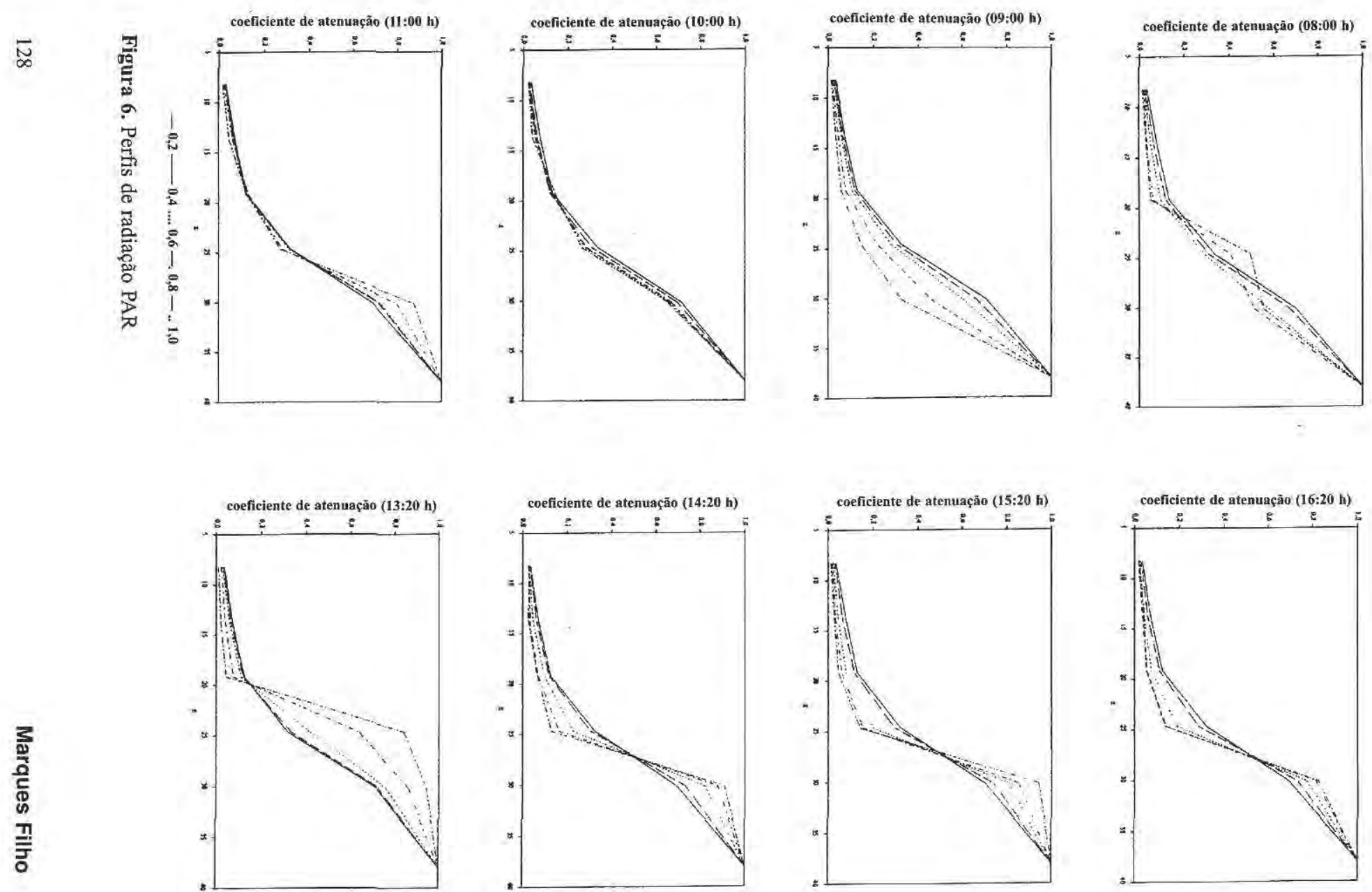
horário correspondente: $<0,2$ (I); 0,2 0,4 (II); $0,4-0,6$ (III); 0,6 - 0,8 (IV); 0,8 - 1,0 (V). Em cada horário e dentro de cada uma das cinco categorias estabelecidas foram calculados perfis de radiação médios e normalizados. Uma visão do comportamento desses perfis e de suas variaçòes temporais é apresentada na figura 6 .

A hora indicada em cada gráfico representa o tempo final do período de 20 minutos adotado para as medidas. Os pares de gráficos dispostos numa mesma posição horizontal são períodos de observações simétricos em relação ao meio-dia ( \pm 4 horas; \pm 3 horas; etc.). Em geral , tanto na parte da manhã como na parte da tarde, as curvas associadas às categorias IV e V (forte contribuição de radiação direta) apresentam um comportamento bem distinto das outras três categorias (predominância de radiação difusa). As irregularidades dos perfis e o afastamento entre as curvas dos dois grupos são evidências das difículdades amostrais para se obter valores médios representativos nas ocorrências de radiação direta em dias com céu limpo ou com pequena cobertura de nuvens. Quando essas duas curvas são retiradas do conjunto de medidas, as restantes apresentam-se entre si de forma bastante concordante e regular, com pequenas dissimetrias entre as medidas da manhã comparadas ao período da tarde, que devem ser atribuidas às características estruturais locais da vegetação. Assumese nas etapas seguintes deste estudo que as curvas pertencentes às categorias I, II e III possam ser descritas adequadamente por um modelo de radiação puramente difuso.

Assim, para essas três categorias e no período diurno entre 7 e 17 horas, foram calculadas as curvas da função A(z) (área foliar acumulada), que resultam da inversão do modelo proposto (equações 2 e 6 ) e em função das medidas de radiação PAR.

$\mathrm{Na}$ figura 7, a mesma visão simétrica em relação ao meio-dia é utilizada para ilustrar os resultados dessa operação. As formas obtidas da função $\mathrm{A}(\mathrm{z})$ são bem concordantes entre as três categorias, as diferenças entre as curvas sendo visualmente comparáveis àquelas das curvas correspondentes às medidas de $\alpha_{1}$. A disposição relativa das curvas em cada gráfico individual respeita a natureza do fenômeno físico em foco e às próprias medidas de radiação: à atenuações maiores do fluxo de radiação ( $\alpha_{\mathrm{t}}$ menor) resultam na inversão, independente das hipóteses e do modelo (supostamente realístico) adotado, valores mais elevados para $A(z)$, Outro aspecto interessante nesses gráficos é a maior coincidência entre as curvas para os horários próximos ao meio-dia, fato que deve ser atribuído em grande parte a maior precisão das medidas para esses horários.

Uma idéia da dispersào dos perfis de $\mathrm{A}(\mathrm{z})$ resultantes da inversão em cada categoria é mostrada na figura 8 (a,b,c), junto com uma visão da dispersão geral de todos os perfis (d). É bastante nítida a pequena variação dos resultados encontrados para as categorias I e II em contraste àqueles da categoria III, que se apresentam com acentuada dispersão: uma ilustração a mais do mesmo problema de amostragem espacial, quando a radiação 

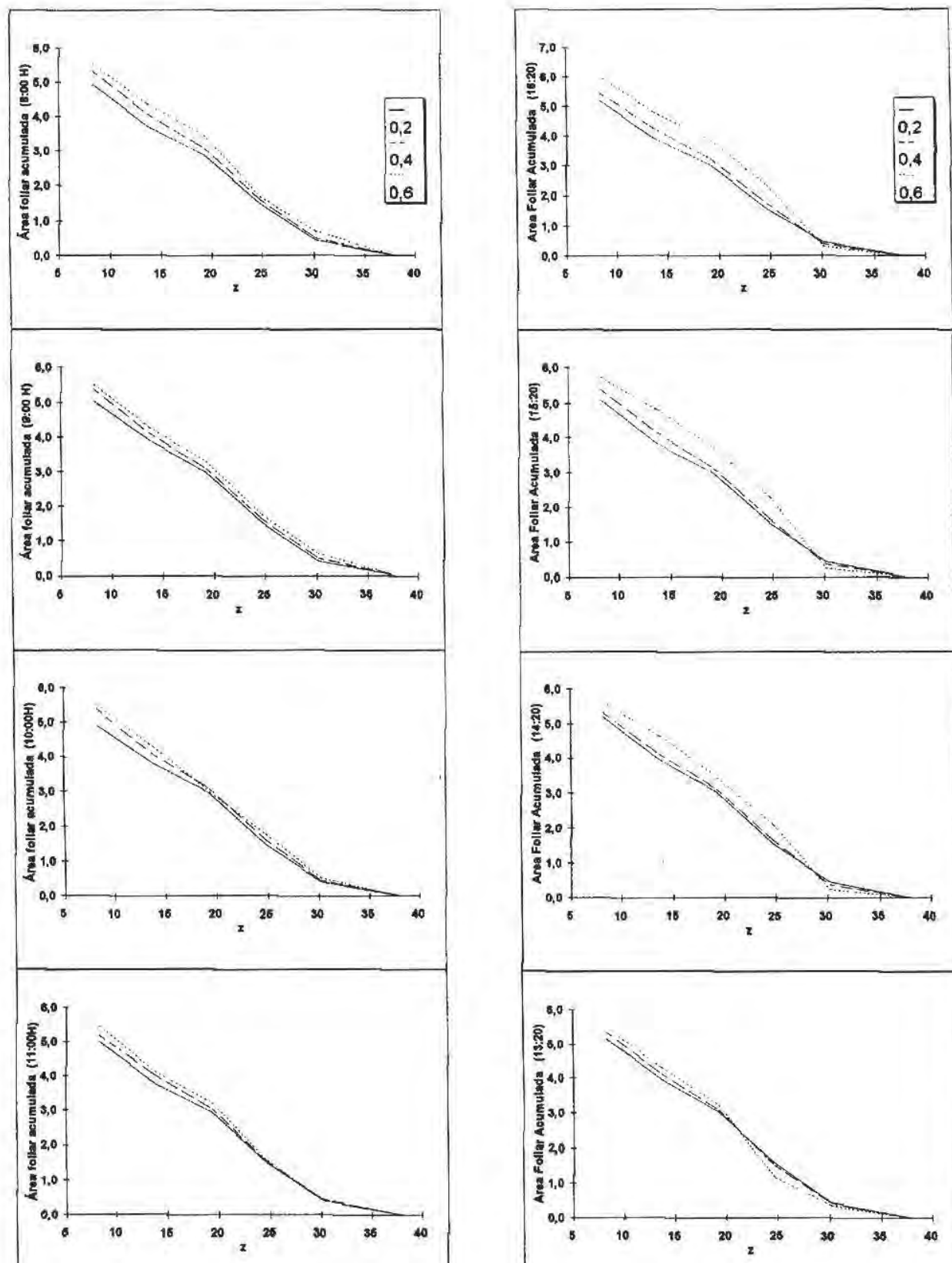

Figura 7. Perfis de Área Foliar Acumulada: $A=A\left(a_{t}\right)$ 


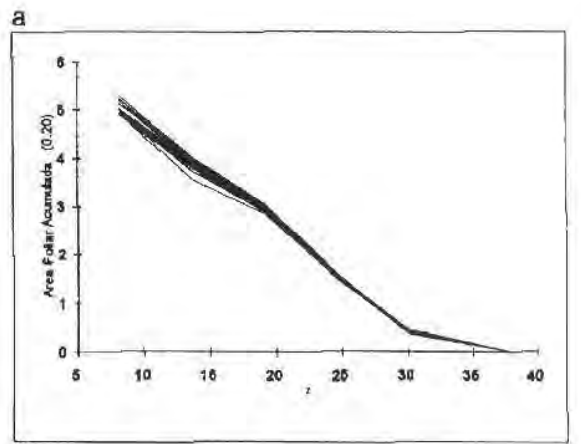

b

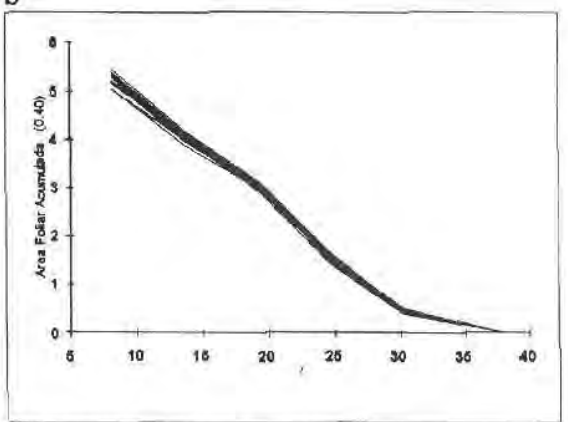

c
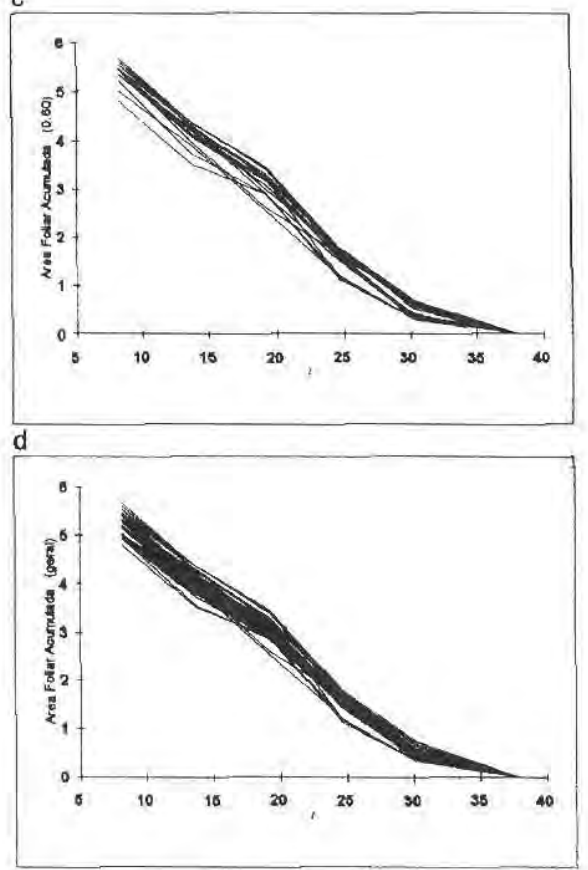

Figura 8. Perfis de A(z)

Tabela 3. Valores obtidos para a função $\mathrm{A}(\mathrm{z})$

\begin{tabular}{c|cccccc}
\hline altura & 8,23 & 13,72 & 19,20 & 24,69 & 30,18 & 38,00 \\
\hline média: I & 5,097 & 3,874 & 2,990 & 1,543 & 0,436 & 0,000 \\
média II & 5,346 & 4,117 & 3,102 & 1,621 & 0,428 & 0,000 \\
média III & 5,545 & 4,364 & 3,332 & 1,780 & 0,428 & 0,000 \\
média geral & 5,329 & 4,118 & 3,141 & 1,648 & 0,431 & 0,000 \\
\hline
\end{tabular}

direta já começa a ter sua influência, ainda que pequena neste caso.

$\mathrm{Na}$ tabela 3 estão reunidos os valores médios da função $A(z)$ para cada uma das categorias (I,II,III) e diferentes niveis no interior da vegetação, além do perfil médio resultante da reunião dos três conjuntos de valores. No nível mais próximo da superficie do solo, a função $\mathrm{A}(\mathrm{z})$ varia na faixa de valores de 5,1 a 5,5 com um valor médio de 5,3; o LAI da floresta neste local deve ser superior a esses resultados, mas da mesma ordem de grandeza.

Esses valores de $\mathrm{A}(\mathrm{z})$ possibilitam o cálculo da função que expressa a densidade de área foliar $a(z)$ para o ponto intermediário $\mathrm{z}_{\mathrm{m}}$ de cada par de valores $A(z)$, ou

$$
a\left(z_{m}\right)=-\frac{d A(z)}{d z}=\frac{A\left(z_{2}\right)-A\left(z_{1}\right)}{z_{1}-z_{2}},
$$

A tabela 4 reúne os valores assim obtidos para a função $a(z)$ e para a área foliar acumulada total da vegetação $A_{0}$ e a figura 9 ilustra a distribuição $\mathrm{a}(\mathrm{z})$ resultante. $\mathrm{O}$ valor máximo de densidade de área foliar situa-se na altura de $22 \mathrm{~m}$ e varia na faixa de 0,26 a 0,28 com um valor médio de 
Tabela 4. Valores obtidos para a densidade de área foliar a(z).

\begin{tabular}{c|cccccccc}
\hline altura & 0,000 & 10,950 & 16,450 & 21,950 & 27,450 & 34,100 & 38,000 & LAI \\
\hline média I & 0,000 & 0,222 & 0,161 & 0,263 & 0,201 & 0,056 & 0,000 & 5,678 \\
média II & 0,000 & 0,223 & 0,185 & 0,269 & 0,217 & 0,055 & 0,000 & 5,941 \\
média III & 0,000 & 0,215 & 0,188 & 0,282 & 0,246 & 0,055 & 0,000 & 6,133 \\
média geral & 0,000 & 0,220 & 0,177 & 0,271 & 0,221 & 0,055 & 0,000 & 5,915 \\
\hline
\end{tabular}

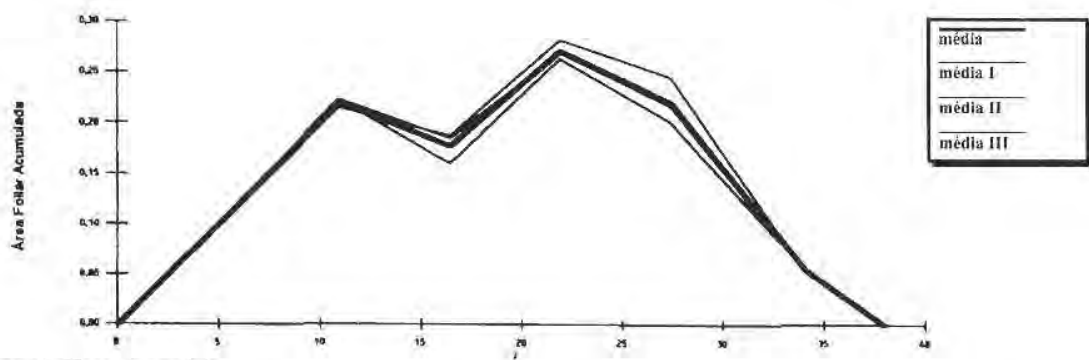

Figura 9. Função $a(z)$

\section{$0,27 \mathrm{~m}^{2} / \mathrm{m}^{3}$. O LAI da vegetação,} implícito nesses cálculos ( integração da área da curva sob a função a(z)), situase entre os limites de 5,7 a 6,1 com um valor médio de 5,9.

\section{CONCLUSÃO}

A utilização de medidas de radiação difusa em florestas para a determinação de características da vegetação (densidade de área foliar, LAI, etc.) pela inversão de modelos teóricos foi abordada neste estudo e o realismo e a estabilidade desse procedimento ficam demonstrados nas formas obtidas para as funções $A(z)$ e $a(z)$. Um maior detalhamento dessas funções é limitado apenas pela necessidade de medidas experimentais adicionais, em que novas configurações e distribuição dos sensores na vertical sejam implementadas; o uso de um grande número de sensores em medidas simultâneas representa o caso ideal para esse tipo de aplicação. No contexto analítico, as equações simplificadas ( 2 e 6 ) que dão o suporte ao presente desenvolvimento são apropriadas para a descrição da radiação PAR, uma análise adicional sendo necessária para a adaptação das equações gerais ao caso de medidas de radiação global (PAR + NIR), A determinação da área foliar acumulada total da vegetação está implícita no procedimento atual e a estimativa próxima de 6 para essa característica da floresta da Reserva Ducke deve ser consistente na faixa de erro de $\pm 10 \%$.

\section{AGRADECIMENTOS}

$\mathrm{O}$ autor agradece aos pesquisadores do Projeto ABRACOS que contribuíram para a realização do presente estudo e em particular aos Drs. John ROBERTS ( Institute of Hydrology) e Osvaldo CABRAL (EMBRAPA) pelas discussões sobre os aspectos teóricos e experimentais do tema 
desenvolvido; aos técnicos da Coordenação de Pesquísas em Geociências do INPA: Afonso Ligório da Mota, Hermes Braga Xavier, Jauapery Neves Pereira Júnior, Sérgio Magno Valério de Souza e Valdelice Pereira da Silva, e estagiários da Universidade do Amazonas: Jean Dalmo de Oliveira Marques e Jânia Lilia Bentes da Silva pela preparação, montagem e execução das medidas na Reserva Florestal Ducke.

\section{Bibliografia citada}

ABRAMOWITZ, M.; STEGUN, 1., 1970 - A Handbook of Mathematical Functions. Dover Publications Washington D.C. 1046 pgs.
ANDRIEU, B.; BARET, F. 1993. Indirects methods of estimating crop structure from optical measurements. In: Crop structure and Light Microclimate, ed. Varlet-Grancher, C.; Bonhomme, R.; Sinoquet, H.: 285-322.

MARQUES FILHO, A.O, 1992a. Modéles des transferts radiatifs à $\mathrm{I}$ interieur des couverts végétaux - les solutions analytiques. Acta Amazonica, 22(4): 541-565.

MYNENI, R.R.; ROSS, J.; ASRAR, G. 1989 ,"A Review on the Theory of Photon Transport in Plant Canopies" - Agricultoral and Forest Meteorology 45: 1-153.

ROSS, J. 1981 - The Radiation Regime and the Architecture of Plant Stands. Dr. W. Junk Publ., The Netherlands. 